\title{
RESPIRACIÓN POTENCIAL DURANTE LA BIOESTIMULACIÓN DE UN SUELO CONTAMINADO CON HIDROCARBUROS AROMÁTICOS POLICÍCLICOS
}

\author{
Silvana Irene TORRI ${ }^{1}{ }^{*}$, Marisol Natalia CABRERA ${ }^{2}$ y Cecilia ALBERTI ${ }^{3}$
}

${ }^{1}$ Departamento de Recursos Naturales y Ambiente, Facultad de Agronomía, Universidad de Buenos Aires. Avenida San Martín 4453, C1417DSE, Buenos Aires, Argentina

${ }^{2}$ Facultad de Ciencias Exactas y Naturales, Universidad de Buenos Aires. Intendente Güiraldes 2160, Ciudad Universitaria, C1428EGA, Buenos Aires, Argentina

${ }^{3}$ Instituto Nacional de Tecnología Industrial-Química. Avenida General Paz 5445, San Martín, B1650 WAB, Buenos Aires, Argentina

*Autor para correspondencia: torri@agro.uba.ar

(Recibido mayo 2016; aceptado junio 2017)

Palabras clave: biodegradación, antraceno, fenantreno, microorganismos, suelos, HAP

\section{RESUMEN}

Los hidrocarburos aromáticos policíclicos (HAP) son compuestos orgánicos que pueden persistir en el ambiente debido a su hidrofobicidad y a su baja solubilidad en agua, lo que representa un riesgo de ingresar a la cadena trófica. Ciertos microorganismos edáficos son potencialmente capaces de degradarlos. La incorporación de nutrientes puede acelerar la tasa de biodegradación si las condiciones del suelo son favorables. Los objetivos del presente trabajo fueron estudiar el efecto disipador de los microorganismos edáficos nativos en un suelo contaminado artificialmente con dos HAP (antraceno o fenantreno), además de evaluar el efecto en la disponibilidad de macro y micro nutrientes. Adicionalmente, se estudió la influencia de la estructura química del contaminante en el proceso de biorremediación. Se realizó un ensayo de incubación con un suelo hapludol típico contaminado artificialmente con antraceno o con fenantreno. $\mathrm{La}$ actividad microbiana se estimó en forma indirecta través de la producción de $\mathrm{CO}_{2}$. La presencia de antraceno o de fenantreno no afectó la actividad microbiana edáfica inicial. Se observó un incremento en la emisión total de $\mathrm{CO}_{2}$ en los suelos contaminados comparado con los suelos sin contaminar debido a la degradación de los contaminantes. Al término de 60 días se analizaron los suelos por cromatografía gaseosa con detector de espectrometría de masas. Según los tratamientos, la concentración de antraceno y de fenantreno disminuyó un $75-77 \%$ o un 89 - $91 \%$, respectivamente. Lo que indica la capacidad de los microorganismos nativos edáficos para degradar rápidamente ambos contaminantes. La disipación del fenantreno fue mayor que la correspondiente al antraceno, lo que fue atribuido a su diferente biodisponibilidad en el suelo. No se observaron diferencias significativas entre tratamientos con y sin nutrientes, por lo que se concluye que la disponibilidad de éstos no fue limitante para la actividad catabólica de los microorganismos edáficos.

Key words: biodegradation, anthracene, phenanthrene, miroorganisms, soil, PAHs 


\begin{abstract}
Polycyclic aromatic hydrocarbons (PAHs) are organic compounds that may persist in the environment due to their hydrophobicity and insolubility in water, posing the risk of entering the food chain. Some soil microorganisms are potentially capable of degrading PAHs. The addition of nutrients may accelerate the rate of biodegradation if soil conditions are favorable. The objectives were to study the degradation capacity of native soil microorganisms to degrade selected PAHs in an artificially contaminated soil, and to evaluate the effect of nutrient availability. The influence of the chemical structure of the contaminant was also studied. An incubation assay was performed, using a typic hapludoll soil artificially contaminated with anthracene or phenanthrene. Microbial activity was indirectly estimated through the production of $\mathrm{CO}_{2}$. Soil contamination with anthracene or phenanthrene did not affect the initial soil microbial activity. The total emission of $\mathrm{CO}_{2}$ increased in contaminated soils compared to the pristine ones, due to the degradation of contaminants. At 60 days, soil samples were analyzed by gas chromatography-mass spectrometry. The removal of anthracene and phenanthrene from the soil was different, and it was related to bioavailability. Anthracene and phenanthrene concentration decreased $75-77 \%$ or $89-91 \%$, respectively, indicating the ability of native soil microorganisms to rapidly degrade both pollutants. No significant differences were observed between treatments with and without nutrients, concluding that nutrient availability was not a limiting factor for the catabolic activity of soil microorganisms.
\end{abstract}

\section{INTRODUCCIÓN}

Los hidrocarburos aromáticos policíclicos (HAP) son contaminantes orgánicos con características ecotoxicológicas, mutagénicas y carcinogénicas ( $\mathrm{Fu}$ et al. 2012, Singh y Gupta 2016). Muchos de ellos son considerados compuestos orgánicos persistentes debido a su estabilidad estructural, hidrofobicidad y baja solubilidad en agua (Cerniglia 1992, Zielińska y Oleszczuk 2016). Si bien el suelo siempre ha sido considerado un sistema depurador, en numerosas ocasiones la velocidad con que los contaminantes ingresan al mismo, supera su capacidad de disipación. De este modo, ciertos contaminantes se acumulan en los ecosistemas, lo que origina efectos negativos en el ambiente (Eom et al. 2007, Wang et al. 2016).

En los suelos, los HAP pueden provocar la inhibición en el crecimiento de los cultivos (Somtrakoon y Chouychai 2013). Ciertos compuestos persistentes consiguen ser absorbidos por las raíces de las especies vegetales, posteriormente son transportados a su parte aérea (Gao y Ling 2006) y con ello, ingresan así a la cadena trófica (de Lima et al. 2015).

La biorremediación se basa en la transformación química de contaminantes mediante el uso de microorganismos que satisfacen sus requerimientos nutricionales y energéticos con dichos contaminantes, contribuyendo a la disipación de HAP por mineralización o co-metabolismo (Cerqueira et al. 2014, Singh y Gupta 2016). Los microorganismos que intervienen en este proceso son principalmente bacterias y en menor proporción, hongos nativos y algas. La tasa de degradación microbiana está condicionada por la capacidad de adaptación de los microorganismos nativos a las nuevas condiciones edáficas, la disponibilidad de nutrientes, el contenido de oxígeno, la temperatura, la conductividad eléctrica (CE) y el pH (Abed et al. 2015, Bansal y Kim 2015, Gupta et al. 2015). La bioestimulación por otro lado, consiste en la incorporación de nutrientes y aceptores de electrones para incrementar la actividad microbiana, con el objeto de acelerar la tasa de biodegradación si las condiciones ambientales son favorables (Tyagi et al. 2011, Wu et al. 2016).

La actividad microbiana es muy sensible a cualquier cambio edáfico (Welp y Brümmer 1999). Su estudio permite analizar el impacto de los contaminantes sobre las comunidades microbianas presentes, estimar la degradación del contaminante y determinar la factibilidad del tratamiento de biorremediación (de Souza Pohren et al. 2016). La respiración es uno de los parámetros más frecuentemente utilizados para cuantificar en forma indirecta la actividad microbiana del suelo (Celis et al. 2009).

El antraceno y el fenantreno son isómeros formados por tres anillos bencénicos fusionados (Fig. 1). Estos isómeros están ampliamente distribuidos en el ambiente y han sido incluido en el listado de los 17 presuntos HAP de ser los más dañinos y con efectos nocivos para los seres humanos (Chirakkara y Reddy 2015, Dubrovskaya et al. 2016). También son utilizados para detectar procesos de contaminación, 


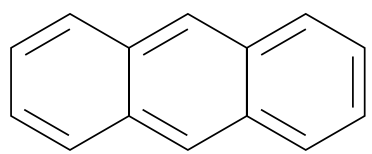

antraceno

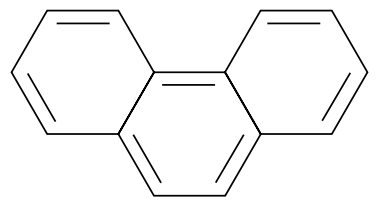

fenantreno
Fig. 1. Estructura del antraceno y del fenantreno

ya que son productos intermedios en la degradación de otros HAP como benzo[a]pireno y benz[a]antraceno (Kanaly Harayama 2000). Debido a que su mecanismo de oxidación es muy similar al de otros HAP más complejos, el antraceno y el fenantreno suelen utilizarse como compuestos poliaromáticos modelo para determinar los factores que afectan la biodisponibilidad, el potencial de biodegradación y la velocidad de degradación microbiana de otros HAP en el ambiente (Kanaly y Harayama 2000).

Los objetivos del presente trabajo fueron: 1) estudiar el efecto disipador de los microorganismos edáficos nativos en un suelo contaminado artificialmente con antraceno o fenantreno, 2) evaluar el efecto de la disponibilidad de nutrientes y 3) analizar la influencia de la estructura química del contaminante en el proceso de biorremediación.

\section{MATERIALES Y MÉTODOS}

Se realizó un ensayo de incubación en la Facultad de Agronomía de la Universidad de Buenos Aires, Argentina. Para ello, se utilizó el horizonte superficial $(0-15 \mathrm{~cm})$ de un suelo hapludol típico (US Soil Taxonomy, USDA, 1999), de la localidad de Carlos Casares $\left(35^{\circ} 37^{\prime} \mathrm{S}-61^{\circ} 22^{\prime} \mathrm{W}\right)$, provincia de Buenos Aires. Se tomaron muestras compuestas de suelos no cultivados $(\mathrm{n}=10)$ con implementos adecuados (pala de acero inoxidable, cuchillo de teflón, tamiz de acero inoxidable) para evitar su contaminación. Dichas muestras se secaron al aire, se molieron y se tamizaron $(<2 \mathrm{~mm})$. Una parte se reservó para determinaciones físicas y químicas.

El suelo se contaminó artificialmente con antraceno o fenantreno. Ambos contaminantes, marca Fluka de laboratorios Sigma-Aldrich, presentaron un valor de pureza superior a $98 \%$. Dada la baja solubilidad de los contaminantes en agua, numerosos trabajos solubilizan los HAP en diferentes solventes orgánicos como acetona o diclorometano, entre otros. Para evitar el daño a la microflora edáfica que ocasiona la presencia de dichos solventes en el suelo (Brinch et al. 2002), los contaminantes se incorporaron en forma de cristales, a razón de $1 \mathrm{~g}$ por kg de suelo (base seca), garantizando una buena homogeneización (Ruberto et al. 2006).

Se preparó una solución nutritiva con un fertilizante de grado equivalente 15-15-15 y micronutrientes. Se utilizaron $250 \mathrm{~g}$ de suelo contaminado con antraceno o fenantreno para los siguientes tratamientos: 1) $\mathrm{T}$ (suelo testigo sin contaminar), 2) $\mathrm{T}+\mathrm{N}$ ( $\mathrm{T}$ con la incorporación de nutrientes), 3) A (suelo contaminado con antraceno), 4) A + N (A con la incorporación de nutrientes), 5) $\mathrm{F}$ (suelo contaminado con fenantreno), 6) $\mathrm{F}+\mathrm{N}$ (F con la incorporación de nutrientes).

Se realizaron cuatro repeticiones por tratamiento. Las muestras de suelo se colocaron en recipientes de vidrio con tapa rosca y se humedecieron a $80 \%$ de capacidad de campo con agua destilada o con solución nutritiva. Las muestras de suelo se mantuvieron a temperatura de laboratorio $\left(25^{\circ} \mathrm{C} \pm 2{ }^{\circ} \mathrm{C}\right)$ durante 60 días, con humedad constante a lo largo del ensayo.

\section{Determinaciones analíticas Respiración}

En cada recipiente se cuantificó la actividad heterótrofa de los microorganismos a través de la emisión de $\mathrm{CO}_{2}$ en forma periódica a lo largo del ensayo. $\mathrm{El} \mathrm{CO}_{2}$ generado por la actividad microbiana se determinó con la técnica de incubación estática, la cual consiste en capturar el $\mathrm{CO}_{2}$ producto de la degradación microbiana con una solución alcalina de $\mathrm{NaOH}$ (Alef y Nannipieri 1995). Posteriormente se cuantificó el $\mathrm{NaOH}$ que no reaccionó mediante titulación por retorno con $\mathrm{HCl}$. Las determinaciones se corrigieron a través de un testigo abiótico (Miles y Doucette 2001).

\section{Antraceno y fenantreno remanente}

Al finalizar el ensayo (día 60) las muestras se extrajeron por ultrasonido con hexano: acetona (3:2, $\mathrm{v} / \mathrm{v})$, según la metodología descripta por Torri et al. (2008). Se pesaron $5 \mathrm{~g}$ de suelo y se agregaron $5 \mathrm{~g}$ de $\mathrm{Na}_{2} \mathrm{SO}_{4}$ anhidro. Se colocaron en tubos de centrifuga y se adicionaron $10 \mathrm{~mL}$ de la mezcla de solventes, se aplicó ultrasonido durante $30 \mathrm{~h}$, se centrifugaron a $1500 \mathrm{rpm}$. El sobrenadante se colocó en un matraz aforado de $250 \mathrm{~mL}$ y se repitieron estos últimos pasos (extracción y centrifugación) dos veces más. Los extractos se destilaron con vacío en un rotavapor (Büchi, Suiza). El extracto obtenido se disolvió nuevamente con ultrasonido y se llevó al volumen de un matraz aforado de $5 \mathrm{~mL}$.

Todas las muestras se analizaron por cromatografía de gases acoplada a un espectrómetro de masas (GC-MS, por sus siglas en inglés), con un cromatógrafo Shimadzu GC-14 (Kyoto, Japon) y un 
espectrómetro modelo HP5989. Se utilizó una columna capilar HP - 5 (30 m largo $\times 0.25$ mm diámetro, $0.25 \mu \mathrm{m}$ grosor de película). Las condiciones fueron, columna: HP - PONA, temperatura de horno: isoterma $90{ }^{\circ} \mathrm{C}$ durante $1 \mathrm{~min}$ y luego rampa de un grado por min hasta $280{ }^{\circ} \mathrm{C}$, flujo de columna $1.43 \mathrm{~mL} / \mathrm{min}$, con inyección con división de flujo (split): 80, en modo de inyección de flujo sin división (splitless); temperatura de Inyección: $250{ }^{\circ} \mathrm{C}$. La temperatura de la cámara de ionización del detector de masas fue de $250{ }^{\circ} \mathrm{C}$. El volumen de inyección fue de $1 \mu \mathrm{L}$. La cuantificación se realizó mediante un estándar externo.

\section{Análisis de resultados}

Los resultados del ensayo de respiración se expresaron como $\mathrm{mg} \mathrm{C}-\mathrm{CO}_{2} / \mathrm{g}$ suelo en base peso seco y se realizó un análisis de varianza, previa comprobación de homocedasticidad de varianzas (prueba de Bartlett) y de normalidad de datos (prueba de Shapiro-Wilk). En caso de no cumplirse la homogeneidad de varianza, las variables fueron usando logaritmo neperiano (ln) para su análisis estadístico (Kuehl 1994). Las medias se analizaron mediante la prueba de la diferencia honestamente significativa (HSD, por sus siglas en inglés) de Tukey, con el nivel de significancia de $p=0.05$. Se utilizó el programa Statistics (versión 1.0, 1996) para dichos análisis.

\section{RESULTADOS}

El suelo presentó las siguientes características iniciales: $\mathrm{pH}=5.12$, carbono orgánico $=28.6 \mathrm{~g} / \mathrm{kg}$, capacidad de intercambio catiónico $(\mathrm{CIC})=22.3 \mathrm{cmol}(\mathrm{c}) /$ $\mathrm{kg}, \mathrm{CE}=0.61 \mathrm{dS} / \mathrm{m}$, fósforo extractable (según Bray y Kurtz 1945) $=10.2 \mathrm{mg} / \mathrm{k}$, nitrógeno total $=2.62 \mathrm{mg} / \mathrm{g}$, arena $=57.6 \%$, limo $=23.2 \%$ y arcilla $=19.2 \%$.

\section{Respiración \\ Suelos contaminados con antraceno}

La producción de $\mathrm{C}-\mathrm{CO}_{2}$ en los suelos incubados se incrementó desde el inicio del ensayo hasta el día 10 , sin observarse diferencias significativas entre tratamientos (Fig. 2a). A partir de día 10, la respiración media en los suelos sin contaminar, descendió hasta llegar a un mínimo en el día 24. Por el contrario, en los suelos contaminados con antraceno, la emisión de $\mathrm{C}-\mathrm{CO}_{2}$ fue la máxima entre los días 10 - 18, con una posterior disminución, llegando a un mínimo en el día 24. A los 31 días se observó un pulso de emisión de $\mathrm{C}-\mathrm{CO}_{2}$ de distinta magnitud en todos los tratamientos.
En la figura 2b se observa un aumento no lineal en la producción acumulada de $\mathrm{C}-\mathrm{CO}_{2}$ en los distintos tratamientos. A partir de día 18 y hasta el final del ensayo, se observan diferencias significativas entre tratamientos. La producción acumulada de $\mathrm{C}-\mathrm{CO}_{2}$ fue significativamente más elevada en los suelos contaminados comparado con los suelos sin contaminar. A partir de día 50, la producción de $\mathrm{C}-\mathrm{CO}_{2}$ acumulada se mantuvo constante. No se observó efecto de la incorporación de nutrientes.

\section{Suelos contaminados con fenantreno}

La producción de $\mathrm{CO}_{2}$ en los suelos incubados se incrementó desde el inicio del ensayo hasta el día

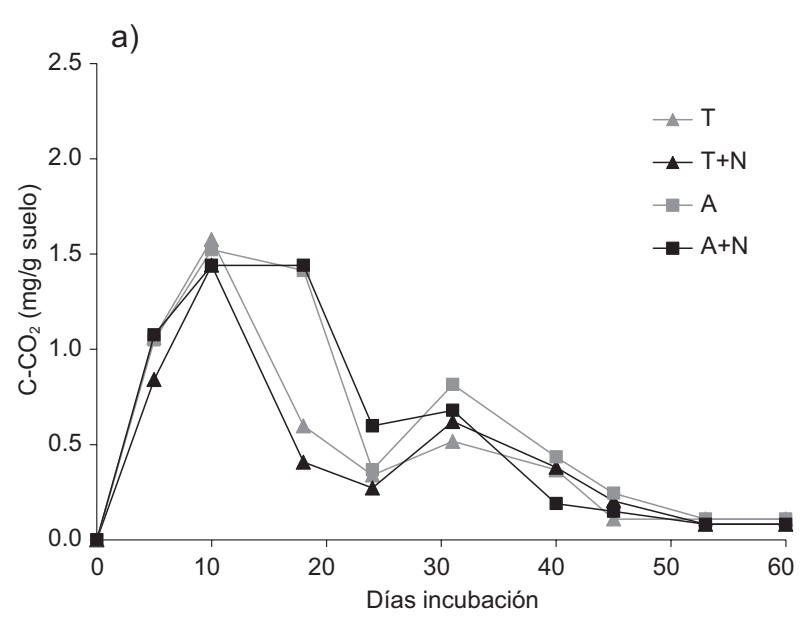

b)

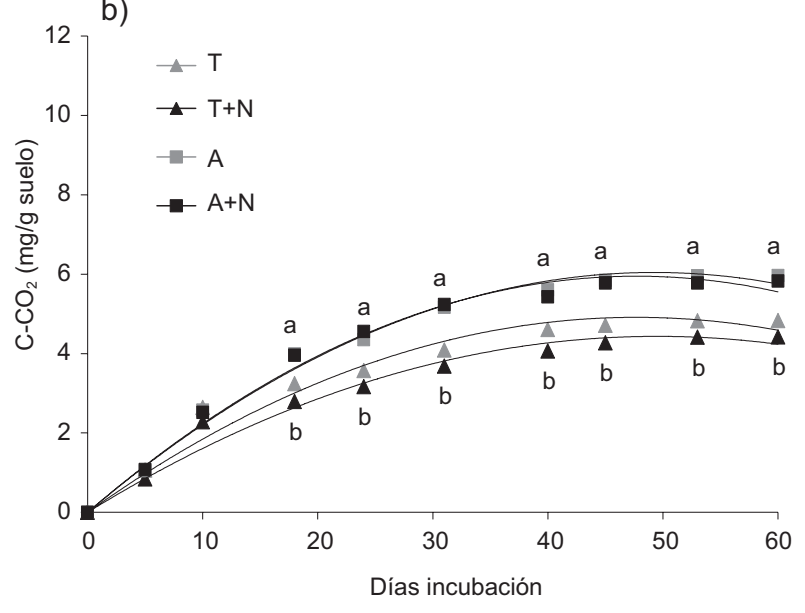

Fig. 2. a) respiración media en cada una de las fechas de muestreo, b) respiración acumulada durante la incubación muestras superficiales $(0-20 \mathrm{~cm})$ perturbadas de un suelo hapludol típico para los siguientes tratamientos: $\mathrm{T}$ (suelo testigo sin contaminar), $\mathrm{T}+\mathrm{N}$ ( $\mathrm{T}$ con la incorporación de nutrientes), $\mathrm{A}$ (suelo contaminado con antraceno), $\mathrm{A}+\mathrm{N}$ (A con la incorporación de nutrientes). Letras distintas para una misma fecha indican diferencias significativas $(\mathrm{p}=0.05)$ 
10 en los suelos sin contaminar y hasta día 18 en los suelos contaminados con fenantreno. Se observaron diferencias significativas entre los días 10 y 45 entre los tratamientos con suelo contaminado y sin contaminar (Fig. 3a). No se observó un efecto en la incorporación de nutrientes.

En la figura 3b se observa un aumento no lineal en la producción acumulada de $\mathrm{C}-\mathrm{CO}_{2}$ en los distintos tratamientos. A partir de día 18 y hasta el final del ensayo se observaron diferencias significativas entre la producción acumulada de $\mathrm{C}-\mathrm{CO}_{2}$ de los suelos contaminados y sin contaminar, la cual resultó ser más elevada en los suelos contaminados. No se ob-

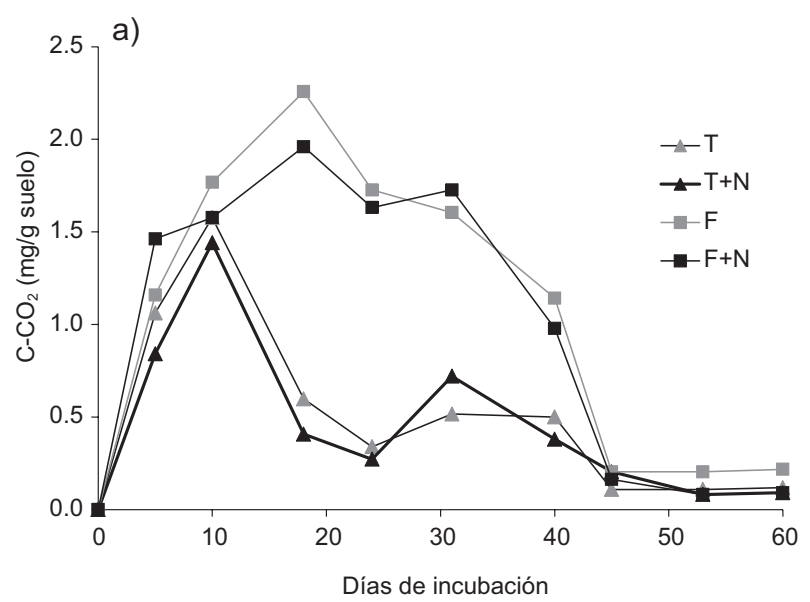

b)

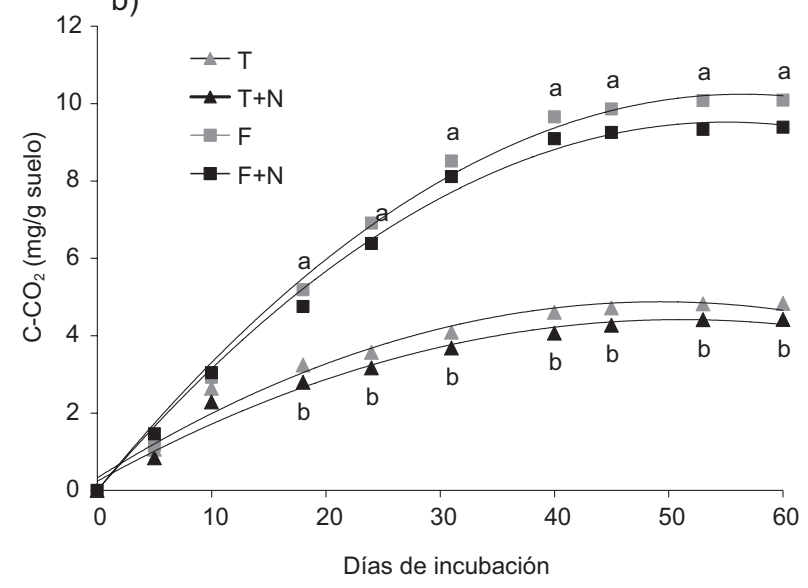

Fig. 3. a) respiración media en cada una de las fechas de muestreo, b) respiración acumulada durante la incubación muestras superficiales $(0-20 \mathrm{~cm})$ perturbadas de un suelo hapludol típico para los siguientes tratamientos: $T$ (suelo testigo sin contaminar), $\mathrm{T}+\mathrm{N}$ ( $\mathrm{T}$ con la incorporación de nutrientes), $\mathrm{F}$ (suelo contaminado con fenantreno), $\mathrm{F}+$ $\mathrm{N}$ (F con la incorporación de nutrientes). letras distintas para una misma fecha indican diferencias significativas $(\mathrm{p}=0.05)$ tuvo un efecto en la incorporación de nutrientes. En contaste con los suelos contaminados con antraceno, los suelos contaminados con fenantreno presentaron una producción acumulada de $\mathrm{C}-\mathrm{CO}_{2}$ significativamente superior.

\section{Antraceno y fenantreno remanente}

El porcentaje de antraceno degradado al final del ensayo fue $75.27 \%$ en el tratamiento A y $77.14 \%$ en el tratamiento $\mathrm{A}+\mathrm{N}$, no se observaron diferencias significativas entre ambos. En el caso del fenantreno, el porcentaje degradado al final del ensayo fue significativamente mayor que el correspondiente a antraceno, con valores de $91.16 \%$ para el tratamiento F y $89.3 \%$ para el tratamiento $\mathrm{F}+\mathrm{N}$, aunque no hubo diferencias significativas entre ambos. En las figuras 4 y 5 se representa el perfil cromatográfico correspondiente a los tratamientos $\mathrm{A}, \mathrm{A}+\mathrm{N}$ y $\mathrm{F}$ y $\mathrm{F}$ $+\mathrm{N}$ al finalizar el periodo de incubación.

\section{DISCUSIÓN}

Los ecosistemas pueden presentar microorganismos heterótrofos nativos potencialmente capaces de utilizar los HAP como fuente de carbono y energía para su crecimiento (Glick 2010, Marco-Urrea et al. 2015). En este trabajo la liberación de $\mathrm{CO}_{2}$ se verificó desde el primer día del ensayo (día 0) en todos los tratamientos. Si bien ciertos trabajos reportan la existencia de una fase inicial, llamada también fase de latencia o adaptación microbiana, que se caracteriza por una mínima liberación de $\mathrm{C}-\mathrm{CO}_{2}$ durante los primeros días en suelos recientemente contaminados con HAP (Reid et al. 2001), la ausencia de dicha fase de adaptación en este ensayo indicaría que ambos contaminantes (antraceno o fenantreno) no originan condiciones de toxicidad para los microorganismos nativos.

La liberación de $\mathrm{C}-\mathrm{CO}_{2}$ en $\mathrm{T}$ y $\mathrm{T}+\mathrm{N}$ hasta el día 10 , corresponde a la mineralización de la materia orgánica nativa del suelo. Los incrementos observados se vinculan con la degradación de las fracciones orgánicas lábiles presentes en el suelo, con la consecuente producción de energía para el crecimiento de los microorganismos. La ausencia de diferencias significativas entre ambos tratamientos indica que, en las condiciones de temperatura y humedad del ensayo, la incorporación de nutrientes no modificó la tasa de liberación de $\mathrm{C}-\mathrm{CO}_{2}$, posiblemente debido a que la disponibilidad de nutrientes era adecuada. Hasta el día 10 no se observaron diferencias significativas entre $\mathrm{T}, \mathrm{T}+\mathrm{N}$, A y A $+\mathrm{N}$, o entre $\mathrm{T}, \mathrm{T}+\mathrm{N}, \mathrm{F}$ 

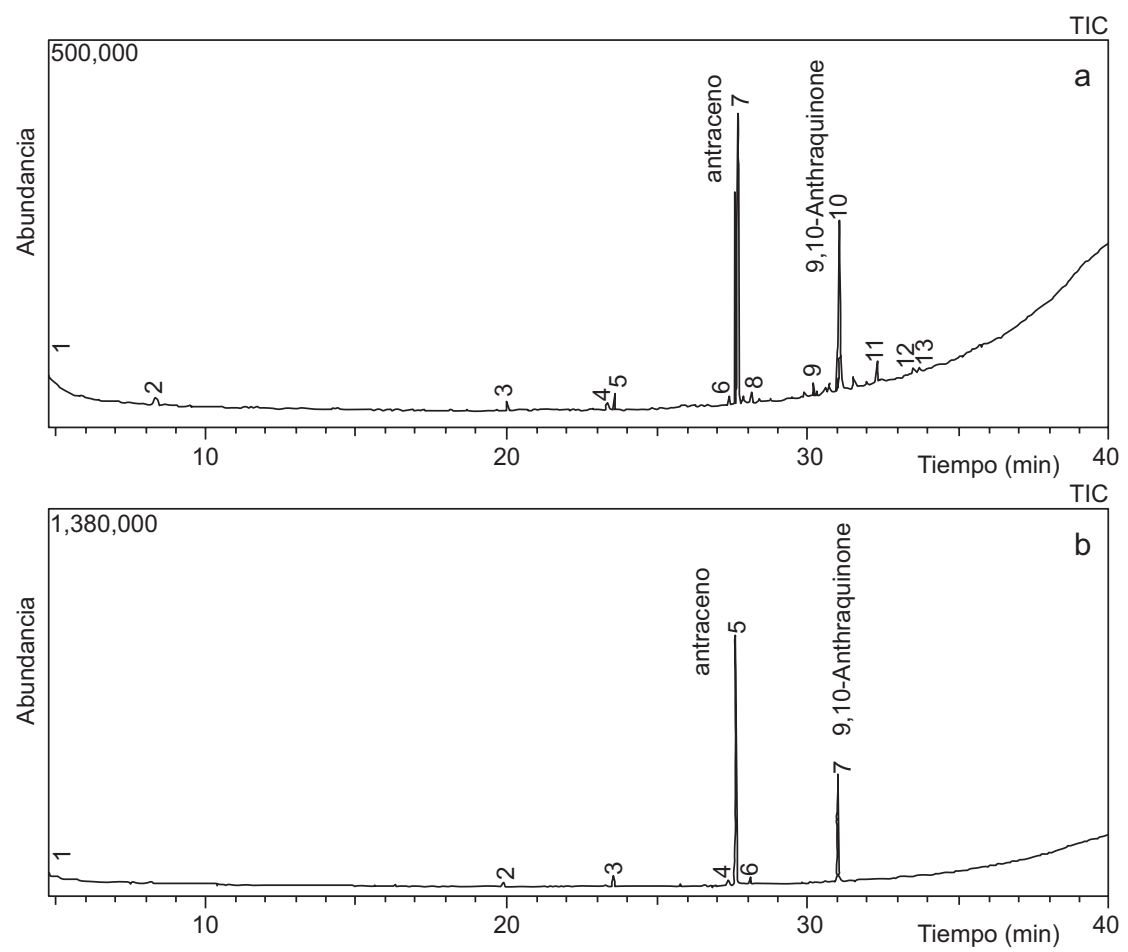

Fig. 4. Perfil de extracción de los metabolitos producidos durante la incubación del suelo con antraceno durante 60 días. a) suelo con incorporación de antraceno, b) suelo con incorporacion de antraceno y nutrientes. TIC $=$ corriente iónica total
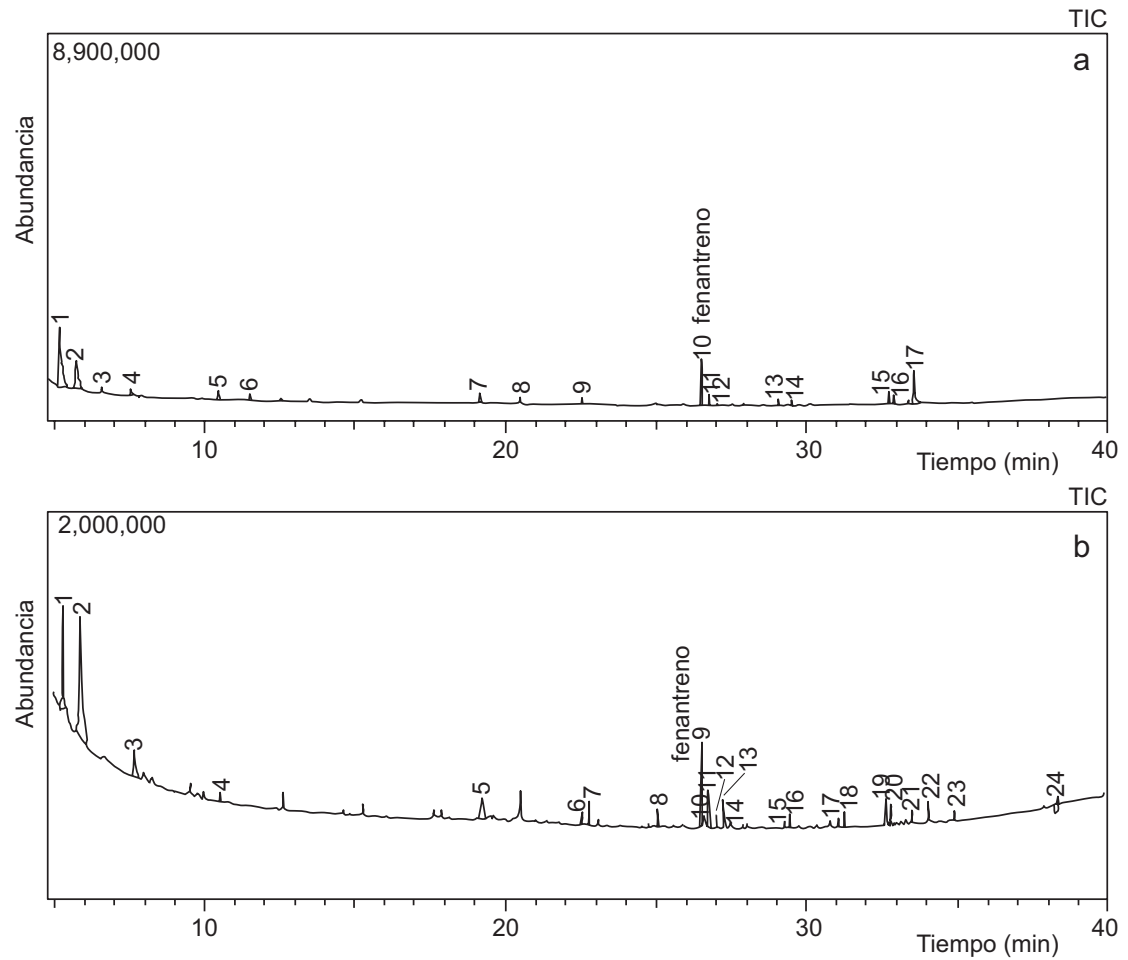

Fig. 5. Perfil de extracción de los metabolitos producidos durante la incubación del suelo con fenantreno durante 60 días. a) suelo con incorporación de fenantreno, b) suelo con incorporacion de fenantreno y nutrientes. TIC $=$ corriente iónica total 
y F $+N$. Estos resultados sugieren que los microorganismos nativos edáficos no degradaron antraceno o fenantreno durante los 10 primeros días y que la contaminación del suelo con ambos contaminantes no alteró la tasa de liberación de $\mathrm{C}-\mathrm{CO}_{2}$ proveniente de la mineralización de la materia orgánica nativa del suelo.

En el día 18 se observó que la emisión de $\mathrm{C}-\mathrm{CO}_{2}$ fue significativamente superior en $\mathrm{A}, \mathrm{A}+\mathrm{N}$ comparado con $\mathrm{T}, \mathrm{T}+\mathrm{N}$. Los mismos resultados se observaron en el caso del fenantreno $(F, F+N)$, lo que sugiere una mayor concentración de materia orgánica fácilmente mineralizable en los suelos contaminados, posiblemente debido a la degradación de antraceno o fenantreno. A pesar de su elevada estabilidad, se han descrito una amplia variedad de microorganismos capaces de degradar compuestos que poseen 1 a 5 anillos aromáticos (Kanaly y Harayama 2000). Ciertos autores indican que los microorganismos utilizan inicialmente sustratos fácilmente degradables como fuente carbonada para metabolizar compuestos de mayor estabilidad que de lo contrario serían poco degradados si estos últimos fueran su única fuente de carbono (Pinto et al. 2009, Mellendorf et al. 2010).

A los 31 días se observó un pulso de emisión de $\mathrm{C}-\mathrm{CO}_{2}$, producto de la utilización de la biomasa microbiana muerta como fuente de carbono por parte de los microorganismos sobrevivientes (Brinch et al. 2002).

En los suelos contaminados con antraceno o fenantreno se observó un aumento no lineal en la producción acumulada de $\mathrm{C}-\mathrm{CO}_{2}$ en los distintos tratamientos a través del tiempo, similar a otro estudio (Guerrero et al. 2007). En todas las curvas se destacan dos etapas (Fig. 2b y 3b): i) una primer etapa de máxima pendiente, con elevada tasa de emisión de $\mathrm{C}-\mathrm{CO}_{2}$ como resultado de una intensa actividad biológica por una mayor disponibilidad de $\mathrm{C}$, energía y nutrientes para los microorganismos heterótrofos del suelo (Zagal et al. 2002, Celis et al. 2009). Esta disponibilidad de energía y nutrientes estimulan tanto el crecimiento como la respiración de los microorganismos y ii) una segunda etapa, de menor pendiente, donde la tasa de emisión de $\mathrm{C}-\mathrm{CO}_{2}$ disminuye con el tiempo como consecuencia de la disminución de sustancias carbonadas fácilmente biodegradables.

La emisión total de $\mathrm{C}-\mathrm{CO}_{2}$ en los suelos contaminados con fenantreno fue significativamente superior que en los suelos contaminados con antraceno. Otros autores también observaron resultados similares (Corona-Ramírez e Iturbe-Argüelles 2005, Fernández-Luqueño et al. 2008). Estos resultados se pueden atribuir a la baja solubilidad del antraceno en sistemas acuosos $(0.07 \mathrm{mg} / \mathrm{L})$ comparado con fenantreno
(1.29 mg/L) (Irwin et al. 1997). Cabe destacar que la solubilidad en agua está directamente relacionada con la biodisponibilidad del contaminante para ser degradado (Abdel-Shafy y Mansour 2016).

Los análisis cuantitativos de los cromatogramas indicaron que los microorganismos nativos presentes en los suelos contaminados degradaron $75.27 \%$ del antraceno en el tratamiento A y $77.14 \%$ del antraceno en el tratamiento $\mathrm{A}+\mathrm{N}$. Otro autor observó una menor degradación de antraceno (2-10\%) en un periodo de tiempo similar (Santos 2004). En las figuras 4 y 5 se aprecia la presencia de 9,10-antraquinona como producto de degradación del antraceno. Debido a su elevada estabilidad química, los anillos aromáticos deben activarse para que puedan ser metabolizados. En la degradación aeróbica del antraceno, las dioxigenasas catalizan la incorporación de dos átomos de oxígeno y dos electrones desde el NADH para formar dihidrodioles. La incorporación de átomos de oxígeno en C-9 y C-10 forma el 9,10 dihidrodiol, que se convierte de modo no enzimático en 9,10-antraquinona, compuesto de elevada estabilidad presente en las muestras incubadas con antraceno.

El porcentaje de fenantreno degradado al final del ensayo fue significativamente superior al de antraceno, lo que corrobora que la mayor tasa de respiración observada en los suelos contaminados con fenantreno se debe a la mineralización de este contaminante. Otros autores también observaron que en suelos contaminados, el fenantreno sufre una mayor mineralización comparado con el antraceno (Jacques et al. 2008). Estos resultados se atribuyeron a la diferente biodisponibilidad edáfica que presentan ambos compuestos (Fernández-Luqueño et al. 2011, Kalantary et al. 2014). El fenantreno presenta una mayor solubilidad en la fase acuosa $(1.9 \mathrm{mg} / \mathrm{L})$ en comparación con el antraceno $(0.07 \mathrm{mg} / \mathrm{L})$, lo que origina una mayor biodisponibilidad por estar en contacto directo con los microorganismos edáficos (Kördel et al. 2013) y así, se facilita su degradación (Mueller y Shann 2006). Se han aislado una gran variedad de microorganismos edáficos con la habilidad de utilizar fenantreno como única fuente carbonada y energética (Wong et al. 2002, Seo et al. 2009).

Tanto el antraceno como el fenantreno remanente en los suelos al final del ensayo (23 - $25 \%$ y $9-11 \%$, respectivamente), parecerían estar en formas no disponibles para los microorganismos edáficos, ya que a partir de día 60 la tasa de liberación de $\mathrm{C}-\mathrm{CO}_{2}$ en los suelos contaminados no se diferenció significativamente de los suelos testigo. Ciertos autores indicaron que la biodisponibilidad de los contaminantes orgánicos disminuye por el tiempo de 
contacto con el suelo (Guo et al. 2016). Este proceso, denominado envejecimiento, está relacionado con la continua difusión de los contaminantes hidrofóbicos hacia el interior de la matriz orgánica o de los micro y nanoporos del suelo, sitios inaccesibles para los microorganismos edáficos. Por lo que no pueden ser metabolizados o transformados en otros compuestos (Semple et al. 2003).

\section{CONCLUSIONES}

Los microorganismos nativos del suelo disiparon la mayor proporción de antraceno y fenantreno en el periodo del ensayo. Según los tratamientos, la concentración de antraceno y fenantreno disminuyó de $75-77 \%$ o de $89-91 \%$, respectivamente, lo que indica la capacidad de los microorganismos para degradar rápidamente ambos contaminantes. El porcentaje de degradación del fenantreno fue significativamente mayor que el del antraceno debido a la mayor solubilidad del primero, que facilita los procesos de degradación microbiana. No se observó un efecto en la incorporación de nutrientes, posiblemente porque su disponibilidad en el suelo era adecuada. Los resultados obtenidos indican que tanto el antraceno como el fenantreno no degradados al finalizar el ensayo se encuentran en formas no disponibles para los microorganismos edáficos.

\section{REFERENCIAS}

Abdel-Shafy H. I. y Mansour M.S.M. (2016). A review on polycyclic aromatic hydrocarbons: Source, environmental impact, effect on human health and remediation. Egypt J. Pet. 25 (1), 107-123.

DOI: $10.1016 /$ j.ejpe.2015.03.011

Abed R. M.M., Al-Kharusi S. y Al-Hinai M. (2015). Effect of biostimulation, temperature and salinity on respiration activities and bacterial community composition in an oil polluted desert soil. Int. Biodeter. Biodegr. 98, 43-52. DOI: 10.1016/j.ibiod.2014.11.018

Alef K. y Nannipieri P. (1995). Methods in applied soil microbiology and biochemistry. Academic Press Limited, San Diego, EUA, 608 pp.

Bansal V. y Kim K. H. (2015). Review of PAH contamination in food products and their health hazards Environ. Int. 84, 26-38. DOI: 10.1016/j.envint.2015.06.016

Brinch U., Ekelund F. y Jacobsen C. (2002). Method for spiking soil samples with organic compounds. Appl. Environ. Microbiol. 68 (4), 1808-1816. DOI: 10.1128/AEM.68.4.1808-1816.2002
Bray R.H. y Kurtz L.T. (1945). Determination of total, organic and available forms of phosphorus in soils. Soil Sci. 59, 39-45.

Celis J., Sandoval M. y Zagal E. (2009). Actividad respiratoria de microorganismos en un suelo patagónico enmendado con lodos salmonícolas. Arch. Med. Vet. 41 (3), 275-279.

DOI: $10.4067 / \mathrm{S} 0301-732 X 2009000300013$

Cerniglia C. E. (1992). Biodegradation of polycyclic aromatic hydrocarbons. Biodegradation 3, 351-368.

Cerqueira V.S., Peralba M.C.R., Camargo F.A.O. y Bento F.M. (2014). Comparison of bioremediation strategies for soil impacted with petrochemical oily sludge. Int. Biodeterior. Biodegrad. 95 (Part B), 338-345. DOI: 10.1016/j.ibiod.2014.08.015

Chirakkara A. y Reddy K.R. (2015). Biomass and chemical amendments for enhanced phytoremediation of mixed contaminated soils. Ecol. Eng. 85, 265-274. DOI: 10.1016/j.ecoleng.2015.09.029

Corona-Ramírez L. e Iturbe-Argüelles R. (2005). Atenuación natural en suelos contaminados con hidrocarburos. Investigación y Tecnología VI (2), 119-126.

de Lima R. F., Dionello R. G., Peralba M. C. R., Barrionuevo S., Radunz L. L. y Reichert Júnior F. W. (2015). PAHs in corn grains submitted to drying with firewood. Food Chem. 215, 165-170.

DOI: 10.1016/j.foodchem.2016.07.164

de Souza Pohren R., de Oliveira Leite D.A.N., de Franceschi de Angelis D. y Vargas V.M.F. (2016). Performance of simulated bioremediation in real samples of soils contaminated with PAHs. Water Air Soil Pollut. 227, 330. DOI: $10.1007 / \mathrm{s} 11270-016-2939-3$

Dubrovskaya E.V., Pozdnyakova N.N., Muratova, Yu A. y Turkovskaya O.V. (2016). Changes in phytotoxicity of polycyclic aromatic hydrocarbons in the course of microbial degradation. Russ. J. Plant Physl. 63 (1), 172-179. DOI: 10.1134/S1021443716010052

Eom I.C., Rast C., Veber A.M. y Vasseur P. (2007). Ecotoxicity of a polycyclic aromatic hydrocarbon (PAH)-contaminated soil. Ecotoxicol. Environ. Saf. 67 (2), 190-205.

DOI: 10.1016/j.ecoenv.2006.12.020

Fernández-Luqueño F., Marsch R., Espinosa-Victoria D., Thalasso F., Hidalgo L. M. E., Munive A., Luna-Guido M. L. y Dendooven L. (2008). Remediation of PAHs in a saline-alkaline soil amended with wastewater sludge and the effect on dynamics of C and N. Sci. Total Environ. 402 (1), 18-28.

DOI: $10.1016 /$ j.scitotenv.2008.04.040

Fernández-Luqueño F., Valenzuela-Encinas C., Marsch R., Martínez Suárez C., Vázquez-Núñez E. y Dendooven L. (2011). Microbial communities to mitigate contamination of PAHs in soil-possibilities and challenges: 
A review. Environ. Sci. Pollut. Res. 18 (1), 12-30. DOI: $10.1007 / \mathrm{s} 11356-010-0371-6$

Fu P.P., Xia Q., Sun X. y Yu H. (2012). Phototoxicity and environmental transformation of polycyclic aromatic hydrocarbons (PAHs) - light-induced reactive oxygen species, lipid peroxidation, and DNA damage J. Environ. Sci. Health Part C. 30 (1), 1-41.

DOI: $10.1080 / 10590501.2012 .653887$

Gao Y. y Ling L. (2006). Comparison for plant uptake of phenanthrene and pyrene from soil and water. Biol. Fertil. Soils 42 (5), 387-394.

DOI: $10.1007 / \mathrm{s} 00374-006-0081-\mathrm{x}$

Glick B.R. (2010). Using soil bacteria to facilitate phytoremediation. Biotechnol. Adv. 28 (3), 367-374.

DOI: $10.1016 /$ j.biotechadv.2010.02.001

Guerrero C., Moral R., Gómez I., Zornoza R. y Arcenegui V. (2007). Microbial biomass and activity of an agricultural soil amended with the solid phase of pig slurries. Biores. Technol. 98 (17), 3259-3264.

DOI: 10.1016/j.biortech.2006.07.015

Guo M., Gong Z., Allinson G., Tai P., Miao R., Li X., Jia C. y Zhuang J. (2016). Variations in the bioavailability of polycyclic aromatic hydrocarbons in industrial and agricultural soils after bioremediation. Chemosphere 144, 1513-1520. DOI: 10.1016/j.chemosphere.2015.10.027

Gupta S., Pathak B. y Fulekar M. H. (2015). Molecular approaches for biodegradation of polycyclic aromatic hydrocarbon compounds: A review. Rev. Environ. Sci. Bio. 14 (2), 241-269.

DOI: $10.1007 / \mathrm{s} 11157-014-9353-3$

Irwin R. J., Van Mouwerik M., Steven L., Seese M. D. y Basham W. (1997). Environmental contaminants encyclopedia. National Park Service, Water Resources Division. Fort Collins, Colorado, EUA, 57 pp.

Jacques R. J. S., Okeke B. C., Bento F. M., Teixeira A. S., Peralba M. C. R. y Camargo F. A. O. (2008). Microbial consortium bioaugmentation of a polycyclic aromatic hydrocarbons contaminated soil Bioresour. Technol. 99 (7), 2637-2643. DOI: 10.1016/j.biortech.2007.04.047

Kalantary R. R., Mohseni-Bandpi A., Esrafili A., Nasseri S., Ashmagh F. R., Jorfi S. y Ja'fari M. (2014). Effectiveness of biostimulation through nutrient content on the bioremediation of phenanthrene contaminated soil.

J. Environ. Health Sci. Eng. 12, 143. DOI: $10.1186 / \mathrm{s} 40201-014-0143-1$

Kanaly R.A. y Harayama S. (2000). Biodegradation of high-molecular-weight polycyclic aromatic hydrocarbons by bacteria. J. Bacteriol. 182 (8), 2059-2067. DOI: $10.1128 / J B .182 .8 .2059-2067.2000$

Kördel W., Bernhardt C., Derz K., Hund-Rinke K., Harmsen J., Peijnenburg W., Comans R. y Terytze K. (2013). Incorporating availability/bioavailability in risk assessment and decision making of polluted sites, using Germany as an example. J. Hazard. Mater. 261, 854-862. DOI: 10.1016/j.jhazmat.2013.05.017

Kuehl R. O. (1994). Diagnosing agreement between the data and the model. Statistical principles of research design and analysis. Duxbury Press, California, EUA, 666 pp.

Marco-Urrea E., García-Romera I. y Aranda E. (2015). Potential of non-ligninolytic fungi in bioremediation of chlorinated and polycyclic aromatic hydrocarbons. New Biotechnol. 32 (6), 620-628.

DOI: $10.1016 /$ j.nbt.2015.01.005

Mellendorf M., Soja G., Gerzabek M. H. y Watzinger A. (2010). Soil microbial community dynamics and phenanthrene degradation as affected by rape oil application. Appl. Soil Ecol. 46 (3), 329-334. DOI: 10.1016/j.apsoil.2010.10.008

Miles R. y Doucette W. (2001). Assessing the aerobic biodegradability of hydrocarbons in two soils using a simple microcosm/respiration method. Chemosphere 45, 1085-1090. DOI: 10.1016/S0045-6535(01)00012-1

Mueller K. E. y Shann J. R. (2006). PAH dissipation in spiked soil: impacts of bioavailability, microbial activity, and trees. Chemosphere 64, 1006-1014.

DOI: 10.1016/j.chemosphere.2005.12.051

Pinto Mariano A., Tomasella R. C., Di Martino C., Maciel Filho R., Regali Seleghim M. H., Contiero J. y de Franceschi de Angelis D. (2009). Aerobic biodegradation of butanol and gasoline blends. Biomass Bioenerg. 33 (9), 1175-1181.

DOI: 10.1016/j.biombioe.2009.05.002

Reid B. J., Mac Leod C.J., Lee P.H., Morriss A.W., Stokes J. D. y Semple K. (2001). A simple ${ }^{14} \mathrm{C}$ respirometric method for assessing microbial catabolic potential and contaminant bioavailability. FEMS Microbiol. Lett. 196 (2), 141-146.

DOI: $10.1111 / \mathrm{j} .1574-6968.2001 . t b 10555 . x$

Ruberto L.A., Vazquez S.C., Curtosi A., Mestre M.C., Pelletier E. y Mac Cormack W.P. (2006). Phenanthrene biodegradation in soils using an Antarctic bacterial consortium. Bioremediat. J. 10 (4), 191-201.

DOI: $10.1080 / 10889860601021449$

Santos E.C. (2004). Anthracene biodegradation stimulated by iron. Tesis de Maestría. Programa de Ciencias del Suelo, Universidad Federal de Rio Grande do Sul, Porto Alegre, Brasil, 230 pp.

Semple K. T., Morriss A. W. J. y Paton G. I. (2003). Bioavailability of hydrophobic organic contaminants in soils: fundamental concepts and techniques for analysis. Eur. J. Soil Sci. 54 (4), 809-818. DOI: $10.1046 /$ j. 1351-0754.2003.0564.X

Seo J. S., Keum Y. S. y Li Q. X. (2009). Bacterial degradation of aromatic compounds. Int. J. Environ. Res. Public Health. 6 (1), 278-309.

DOI: $10.3390 /$ ijerph6010278 
Singh D.K. y Gupta T. (2016) Effect through inhalation on human health of $\mathrm{PM}_{1}$ bound polycyclic aromatic hydrocarbons collected from foggy days in northern part of India. J. Hazard. Mater. 306, 257-268. DOI: 10.1016/j.jhazmat.2015.11.049

Somtrakoon K. y Chouychai W. (2013). Phytotoxicity of single and combined polycyclic aromatic hydrocarbons toward economic crops. Russ. J. Plant Phys. 60 (1), 139-148. DOI: 10.1134/S1021443712060155

Torri S. I., Alberti C., Castelli M. L., Della Vecchia M. y Rossi R. (2008). Caracterización de la materia orgánica del biosólido. Memorias. XXI Congreso Argentino de la Ciencia del Suelo, San Luis. Del 13 al 16 de mayo, 2008. Trabajo completo en CD. ISBN: 978987-21419-9-8.

Tyagi M., da Fonseca M. M. R. y de Carvalho C. C. R. (2011). Bioaugmentation and biostimulation strategies to improve the effectiveness of bioremediation processes. Biodegradation 22 (2), 231-241. DOI: $10.1007 / \mathrm{s} 10532-010-9394-4$

USDA (1999). Soil taxonomy. A basic system of soil classification for making and interpreting soil surveys, $2^{\circ}$ ed. Soil Survey Staff. United States Department of Agriculture, Natural Resources Conservation Service, Agriculture Handbook Number 436, 886 pp.

Wang L., Wang L., Tao W., Smardon R. C., Shi X. y Lu X. (2016). Characteristics, sources, and health risk of polycyclic aromatic hydrocarbons in urban surface dust: a case study of the city of Xi'an in Northwest China. Environ. Sci. Pollut. Res. 23 (13), 13389-13402. DOI: $10.1007 / \mathrm{s} 11356-016-6528-1$

Welp G. y Brümmer G. W. (1999). Effects of organic pollutants on soil microbial activity: influence of sorption, solubility, and speciation. Ecotoxicol. Environ. Saf. 43 (1), 83-90. DOI: 10.1006/eesa.1999.1770

Wong J.W.C., Lai K.M., Wan C.K., Ma K.K. y Fang M. (2002). Isolation and optimization of PAH degradative bacteria from contaminated for PAHs bioremediation. Water Air Soil Poll. 139 (1-4), 1-13.

DOI: 10.1023/A:1015883924901

Wu M., Dick W. A., Li W., Wang X., Yang Q., Wang T., Xu L., Zhang M. y Chen L. (2016). Bioaugmentation and biostimulation of hydrocarbon degradation and the microbial community in a petroleum-contaminated soil. Int. Biodeter. Biodegr. 107, 158-164.

DOI: 10.1016/j.ibiod.2015.11.019

Zagal E., Rodríguez N., Vidal I. y Quezada L. (2002). Actividad microbiana en un suelo de origen volcánico bajo distinto manejo agronómico. Agric. Téc. 62 (2), 297-309. DOI: 10.4067/S0365-28072002000200012

Zielińska A. y Oleszczuk P. (2016). Bioavailability and bioaccessibility of polycyclic aromatic hydrocarbons (PAHs) in historically contaminated soils after lab incubation with sewage sludge-derived biochars. Chemosphere 163, 480-489.

DOI: $10.1016 /$ j.chemosphere.2016.08.072 